\title{
FAUNA EDÁFICA EM ÁREAS COM DIFERENTES MANEJOS E TEMPOS DE DESCARTE DE RESÍDUOS ANIMAIS
}

\author{
Soil fauna in areas with different management and disposal times of animal waste
}

\author{
Luís Carlos Iuñes de Oliveira Filho ${ }^{1 *}$, Luciana Ferro Schneider ${ }^{2}$, Juliano Silva Teles ${ }^{3}$, Silvia Danieli Werter ${ }^{4}$, Julio \\ Cesar Pires Santos ${ }^{5}$
}

\author{
1 Pós-Doutorando, Universidade do Estado de Santa Catarina; iunes1981@gmail.com \\ *Autor para correspondência \\ ${ }^{2}$ Extensionista rural nível superior, Empresa de Pesquisa Agropecuária e Extensão Rural de Santa Catarina; \\ lucianaschneider@epagri.sc.gov.br \\ ${ }^{3}$ Mestrando Ciência do Solo, Universidade do Estado de Santa Catarina; juliano.teles@edu.udesc.br \\ ${ }^{4}$ Graduanda em Agronomia, Universidade do Estado de Santa Catarina; silvia.werter@hotmail.com \\ ${ }^{5}$ Professor, Universidade do Estado de Santa Catarina; julio.santos@udesc.br
}

Artigo enviado em 10/08/2017, aceito em 12/12/2017 e publicado em 10/04/2018.

Resumo - O objetivo deste estudo foi avaliar a presença da fauna edáfica sob diferentes manejos e tempos de descarte de resíduos animais. As áreas utilizadas para o estudo foram diferenciadas pelos seguintes manejos: mata nativa, pastagem nativa sem descarte e as áreas de uso agrícola: pastagem de azevém com 5 e 17 anos de descarte de resíduos animais, milho para silagem com 9 e 22 anos de descarte, pastagem sem pastejo com 22 anos de descarte. Em cada época de coleta foram amostrados 9 pontos por área, com espaçamento de 30 metros entre cada ponto. Foram realizadas avaliações da fauna (método do Berllese e TSBF) e atributos físicos e químicos. Utilizou-se a curva de resposta principal (CRP) para observar o comportamento das áreas ao longo do tempo e análise de redundância (ADR) para os atributos que explicaram a variabilidade dos grupos da fauna edáfica. A área de pastagem com 22 anos se mostrou mais estável em relação as demais (exceto a mata nativa) quanto ao comportamento da densidade dos organismos ao longo do tempo pela CRP. Os atributos $\mathrm{MO}$ e $\mathrm{Cu}$ foram variáveis explicativas significativas na ADR para as duas metodologias considerando todas as épocas de coleta. O descarte continuado de resíduos animais no solo ocasiona mudanças no ambiente influenciando as populações da fauna edáfica.

Palavras-chave - Dejeto de suíno. Cama de aves. Análise multivariada.

Abstract - The goal of this study was to evaluate the presence of soil fauna under different managements and times disposal of animal waste. The areas used for the study were differentiated by the following managements: native forest, native pasture without disposal and areas of agricultural use: ryegrass pasture with 5 and 17 years of disposal of animal waste, corn for silage with 9 and 22 years of disposal pasture without grazing with 22 years of disposal. Each season were sampled 9 points per area, with spacing of 30 meters between each point. Were performed fauna assessments (method Berllese and TSBF) and physical and chemical attributes. We used the principal response curve (PRC) to observe the behavior of the areas over time and redundancy analysis (RDA) for the attributes that explain the variability of the soil fauna groups. The pasture area with 22 years was more stable compared the others (except the native forest) how the behavior of the density of organisms over time by CRP. The OM and $\mathrm{Cu}$ attributes were significant explanatory variables in ADR for the two techniques considering all the collecting seasons. Continued disposal of animal waste in the soil causes changes in the environment influencing the populations of soil fauna.

Keywords - Swine manure. Poultry litters. Multivariate analysis. 


\section{INTRODUÇÃO}

A suinocultura e avicultura são importantes alternativas para incremento da renda no Sul do Brasil. Santa Catarina destaca-se no cenário brasileiro por possuir um dos maiores complexos industriais voltados à cadeia produtiva de suínos, chegando a representar $30 \%$ da produção nacional (ACCS, 2013) e um dos principais produtores nacionais de carne de frango com cerca de $20 \%$ de toda a produção nacional (UBABEF, 2014).

O uso de resíduos animais (dejetos de suínos e cama de aves) como fertilizantes do solo é a forma de reciclagem predominante e mais frequente, devido, esse ser um insumo de baixo custo e com benefício para a fertilidade do solo (MONDARDO et al., 2009; LOURENÇO et al., 2013). Entretanto, o grande volume de dejetos e resíduos produzidos ligado à concentração da produção e descarte em determinadas regiões, de acordo com (KONZEN, 2003), podem ser potencialmente poluidores dos recursos naturais.

A fauna edáfica é componente importante dos ecossistemas nativos e sensíveis às mudanças do habitat (BROMHAM et al., 1999). Além de muito afetada pelas práticas agrícolas, apresenta relação com as características físico-químicas que a torna uma ferramenta na avaliação e monitoramento da qualidade do solo (AQUINO; CORREIA; ALVES, 2008).

A densidade e a diversidade de organismos da fauna do solo, assim como a presença de determinado grupo específico em um sistema, podem ser usadas como indicadores de qualidade dos solos (BARROS et al., 2003). A fauna do solo responde aos impactos causados pelo manejo e constitui-se num bom indicador para avaliações da qualidade dos solos submetidos a diferentes sistemas de manejo (SILVA et al., 2007).

Nesse sentido, a biodiversidade e a complexidade aumentadas sobre o solo contribuem para o restabelecimento ou proteção da multiplicidade de organismos do solo capazes de executar funções biológicas essenciais. Entretanto, os processos de perturbação (por exemplo, aplicação de dejetos e resíduos animais) que afetam essa biodiversidade do solo também se manifestam em diferentes escalas espaciais e temporais, sendo importante o monitoramento dos bioindicadores do solo (SWIFT et al., 2010).

Sabe-se que o descarte de dejeto de suínos e de cama de aves nos solos e seu uso na agricultura pode melhorar a fertilidade do solo e favorecer o crescimento vegetal, mas não é uma prática bem entendida em termos de proteção da biodiversidade no solo. O conhecimento sobre os efeitos desses resíduos nas comunidades da biota do solo para os serviços de ecossistemas é escasso. Assim, o objetivo deste estudo foi avaliar a presença da fauna edáfica em um Nitossolo Vermelho eutrófico cultivado sob diferentes manejos e tempos de descarte de resíduos animais, respondendo as seguintes perguntas: (1) $\mathrm{O}$ descarte continuado de resíduos animais no solo ocasiona mudanças no mesmo, quais atributos que influenciaram os organismos? (2) Quanto mais intensivo for o manejo, menor será a biodiversidade da fauna edáfica ao longo das estações do ano?

\section{MATERIAL E MÉTODOS}

O estudo foi realizado no município de Concórdia SC, em um Nitossolo Vermelho eutrófico (EMBRAPA, 2006), e constou de uma cronossequência de áreas agrícolas em diferentes manejos e tempos de descarte de resíduos animais (5, 9, 17 e 22 anos de uso agrícola, referente a 2011) (Tabela 1). Além dessas áreas, utilizaram-se duas áreas de referência sem descarte de resíduos animais que foram mata nativa (controle) e pastagem naturalizada. O clima da região é classificado segundo Köppen como Cfa, temperado, sem estação seca e verão quente $\left(>22{ }^{\circ} \mathrm{C}\right)$ (PEEL; FINLAYSON; MCMAHON, 2007).

Em cada uma das áreas foi demarcado um grid de nove pontos, com espaçamento de $30 \mathrm{~m}$ entre cada ponto e $20 \mathrm{~m}$ de bordadura, totalizando uma área de um hectare. Cada ponto foi considerado como repetição dentro de cada área estudada. As amostragens foram realizadas em dois anos consecutivos, primavera e dezembro de 2011, outono e inverno de 2012. A amostragem da mesofauna foi realizada nas três primeiras épocas e a macrofauna em todas.

Foram utilizados dois métodos de coleta. A coleta da mesofauna foi feita utilizando-se um cilindro de $10,5 \mathrm{~cm}$ de diâmetro a $10 \mathrm{~cm}$ de profundidade. A extração da mesofauna foi feita através do método de Berllese modificado (SOUTHWOOD, 1968) onde o solo permaneceu por um período de sete dias. A macrofauna edáfica foi coletada através da retirada de monólitos do solo de $25 \times 25 \mathrm{~cm}$ na camada de $0-10 \mathrm{~cm}$ e posterior triagem do monólito e coleta dos organismos visíveis a olho nu (BIGNELL et al., 2010).

A fauna edáfica coletada foi guardada em álcool etílico $(80 \%)$ e analisada na lupa com aumento $40 \times$, onde os grupos foram identificados, quantificados e separados. Os organismos identificados foram: Ac = Acarina; Ara = Araneae; $\mathrm{Chi}=$ Chilopoda; $\mathrm{Col}=$ Coleoptera; Coll = Collembola; Der = Dermaptera; Dipl = Diplura; Diplo $=$ Diplopoda; Dipt $=$ Diptera; Enc $=$ Enchytraeidae; Hemip $=$ Hemiptera; Het $=$ Heteroptera; Hym = Hymenoptera (Formiga); Imaturo = organismos imaturos; Iso = Isopoda; Isop = Isoptera; LDipt $=$ Larva de Diptera; Olig = 
Oligochaeta; Outros = organismos não identificados; Prot $=$ Protura; Pse $=$ Pseudoescorpionida; Sym = Symphyla.

As variáveis explicativas analisadas foram $\mathrm{pH}-\mathrm{H}_{2} \mathrm{O}, \mathrm{Ca}, \mathrm{Mg}, \mathrm{Al}, \mathrm{Na}, \mathrm{P}, \mathrm{K}$, matéria orgânica (MO), Cu, Zn, Fe, Mn, Ca/Mg, CTC efetiva, de acordo com Tedesco et al. (1995), granulometria (GEE; BAUDER, 1986), umidade do solo (Umid), porosidade total $(\mathrm{Pt})$ e macroporosidade (Ma) e microporosidade (Mi) nas camadas de 0-5 e 5-10 cm., de acordo com Embrapa (1997).

Tabela 1. Características das áreas estudadas sob diferentes manejos e tempos de descarte de resíduos animais em um Nitossolo Vermelho eutrófico de Concórdia, SC

\begin{tabular}{|c|c|}
\hline Áreas & Descrição/Histórico \\
\hline $\begin{array}{l}\text { Mata Nativa - MN } \\
27^{\circ} 18^{\prime} 44^{\prime \prime} \mathrm{S} \\
51^{\circ} 59^{\prime} 13^{\prime \prime} \mathrm{W}\end{array}$ & $\begin{array}{l}\text { Área: } 2 \text { ha. Fragmento florestal secundário com pouca interferência antrópica, } \\
\text { porém com ocorrência de espécies exóticas. }\end{array}$ \\
\hline Pastagem naturalizada - P0 & Área: 2 ha. Sem descarte de resíduos de animais e pastejada. \\
\hline $\begin{array}{l}27^{\circ} 19^{\prime} 09^{\prime \prime S} \\
51^{\circ} 00^{\prime} 14^{\prime \prime} \mathrm{W}\end{array}$ & Lotação: 35-40 bovinos de leite na propriedade. \\
\hline $\begin{array}{l}\text { Pastagem } 5 \text { anos - P5 } \\
27^{\circ} 19^{\prime} 06^{\prime \prime S} \\
52^{\circ} 000^{\prime} 13^{\prime \prime w}\end{array}$ & $\begin{array}{l}\text { Área: } 1 \text { hectare. Descarte de dejetos durante } 5 \text { anos a cada } 3 \text { meses; descarte de } \\
\text { cama de aves uma vez ao ano. Cultivo de aveia e azevém no inverno e no verão } \\
\text { milheto e capim Sudão. } \\
\text { Lotação: } 35-40 \text { bovinos de leite na propriedade. }\end{array}$ \\
\hline $\begin{array}{l}\text { Milho } 9 \text { anos - M9 } \\
27^{\circ} 18 \text { '33"S } \\
52^{\circ} 00^{\prime} 10^{\prime \prime W} \\
\text { Pastagem } 17 \text { anos - P17 } \\
27^{\circ} 19^{\prime} 01^{\prime \prime S} \\
52^{\circ} 00^{\prime} 11^{\prime \prime W}\end{array}$ & $\begin{array}{l}\text { Área: } 4 \text { hectares. Duas safras anuais de milho para silagem; } 9 \text { anos de descarte de } \\
\text { dejetos de suínos a cada } 2 \text { meses; descarte de cama de aves ( } 1 \text { vez ao ano). } \\
\text { Semeadura direta e pousio curto ( } 3 \text { meses) no inverno. } \\
\text { Área: } 2 \text { hectares. Descarte de dejetos de suínos durante } 17 \text { anos, a cada } 3 \text { meses; } \\
\text { descarte de cama de aves ( } 1 \text { vez ao ano). Escarificação uma vez no ano, cultivo de } \\
\text { aveia e azevém no inverno e no verão milheto e capim sudão. } \\
\text { Lotação: } 35-40 \text { bovinos de leite na propriedade. }\end{array}$ \\
\hline $\begin{array}{l}\text { Milho } 22 \text { anos - M22 } \\
27^{\circ} 19^{\prime} 16^{\prime \prime} \mathrm{S} \\
52^{\circ} 00^{\prime} 23^{\prime \prime} \mathrm{W}\end{array}$ & $\begin{array}{l}\text { Área: } 3 \text { hectares. Duas safras anuais de cultivo de milho para silagem; } 22 \text { anos de } \\
\text { descarte de dejetos de suínos; descarte de cama de aves ( } 1 \text { vez ao ano). Preparo } \\
\text { convencional do solo; cultivo de azevém no inverno e aplicação de } 5 \text { toneladas ha- } \\
{ }^{1} \text { de calcário realizada em } 2008 \text {. }\end{array}$ \\
\hline $\begin{array}{l}\text { Pastagem } 22 \text { anos - P22 } \\
27^{\circ} 18^{\prime} 45^{\prime \prime S} \\
51^{\circ} 59^{\prime} 32^{\prime \prime W}\end{array}$ & $\begin{array}{l}\text { Área: } 1,5 \text { ha. Descarte de dejetos há } 22 \text { anos, cama de aves uma vez ao ano nos } \\
\text { primeiros } 10 \text { anos e aplicação anual de esterco suíno. Pastagem de azevém, aveia e } \\
\text { nabo forrageiro, sem pastejo e sem preparo do solo. }\end{array}$ \\
\hline
\end{tabular}

Por se tratar de um estudo ecológico e estar localizado em um mesmo tipo de solo, o desenho experimental utilizado no estudo inclui pseudorrepetições (nove), optando por casualizar os pontos de amostragem dentro de cada área e considerando como um delineamento inteiramente casualizado. Esses dados devem ser interpretados assumindo que não há outros fatores que afetam a fauna do solo mais forte do que os diferentes manejos e tempos de descarte de resíduos animais. Outros estudos já adotaram essa abordagem resultando em contribuições para Ciência do Solo (MENYAILO, HUNGATE; ZECH, 2002; CHODAK; NIKLIŃSKA, 2010; ASSIS et al., 2014).

A partir do resultado de densidade de indivíduos (ind. $\mathrm{m}^{-2}$ ), os grupos da fauna edáfica foram utilizados como variáveis respostas (efeito) e os atributos químicos e físicos do solo utilizados como variáveis explicativas (causa) na análise de redundância
(ADR). Realizou-se se uma forward selection usando sucessivas ADR para cada tipo de variável, selecionando as que melhor explicaram a variação dos dados $(\mathrm{p}<0,05)$ permitindo a escolha de um conjunto mínimo de variáveis para explicar a variação dos grupos da fauna do solo em cada época de coleta.

Ainda, utilizou-se a curva de resposta principal (CRP) (VAN DEN BRINK; TER BRAAK, 1999) para avaliar o efeito dos diferentes manejos e tempos de descarte de resíduos animais sobre os grupos da fauna edáfica ao longo do tempo. Comparações entre o controle e tratamentos foram submetidos ao teste Dunnett $(\mathrm{p}<0,05)$. Os dados foram analisados utilizando o Software CANOCO versão 4.5 (Microcomputer Power, Wageningen, Netherlands) (TER BRAAK; ŠMILAUER, 2002). Mais informações sobre a CRP podem ser obtidas em (VAN DEN BRINK; TER BRAAK, 1999; TER BRAAK; 
ŠMILAUER, 2002; OLIVEIRA FILHO; BARETTA; SANTOS, 2014).

\section{RESULTADOS E DISCUSSÃO}

\section{Análise de redundância (ADR) para os grupos da fauna}

Através da análise de redundância (ADR) foi possível decompor a variabilidade dos grupos da fauna edáfica como resposta à variabilidade de alguns atributos químicos e físicos do solo em cada época de amostragem, podendo assim, apontar a relação de ocorrência de um grupo específico da fauna com determinado atributo do solo.

$\mathrm{Na}$ primeira época de coleta (primavera), a ADR do método de Berllese modificado (Figura 1a) indicou correlação significativa (por meio do Teste de Monte Carlo, $\mathrm{F}=4,7 ; \mathrm{p}=0,002)$ com as variáveis ambientais do modelo: $\mathrm{Ca} / \mathrm{Mg}(\mathrm{F}=4,71, \mathrm{p}=0,002)$, $\mathrm{Zn}(\mathrm{F}=1,97, \mathrm{p}=0,014), \mathrm{Mn}(\mathrm{F}=2,14, \mathrm{p}=0,012)$, $\mathrm{MO}(\mathrm{F}=1,80, \mathrm{p}=0,032)$, Umidade $(\mathrm{F}=1,76, \mathrm{p}=$ $0,058)$ e $\mathrm{Ma} 0-5 \quad(\mathrm{~F}=1,65, \mathrm{p}=0,054)$. Não considerando as variáveis ambientais, o eixo 1 explica $10 \%$ do total da variância e o eixo 2 explica $3,8 \%$. As variáveis ambientais explicam $20,7 \%$ do total de variação, deste $48,3 \%$ é explicado pelo eixo 1 e 18,2\% pelo eixo 2 . (a)

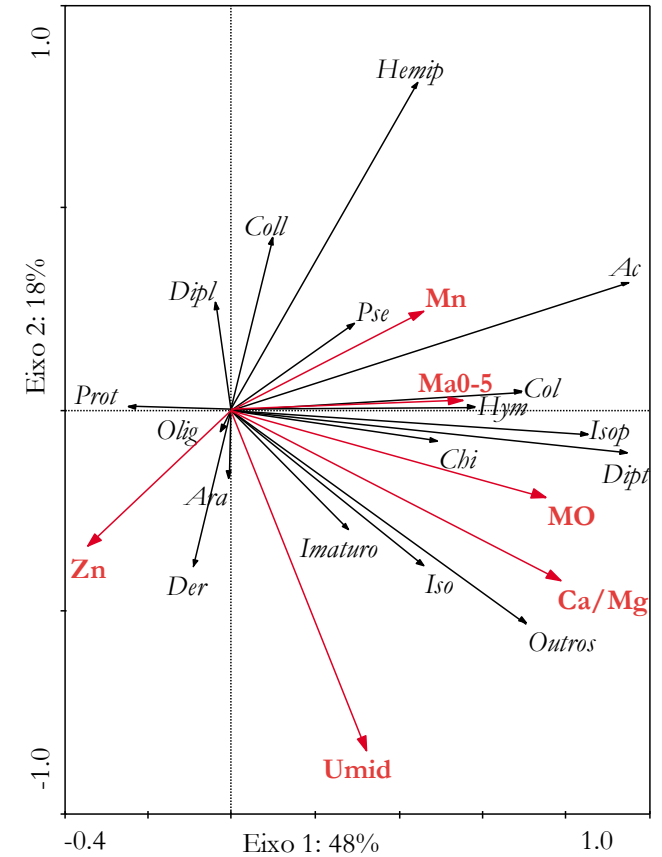

(b)

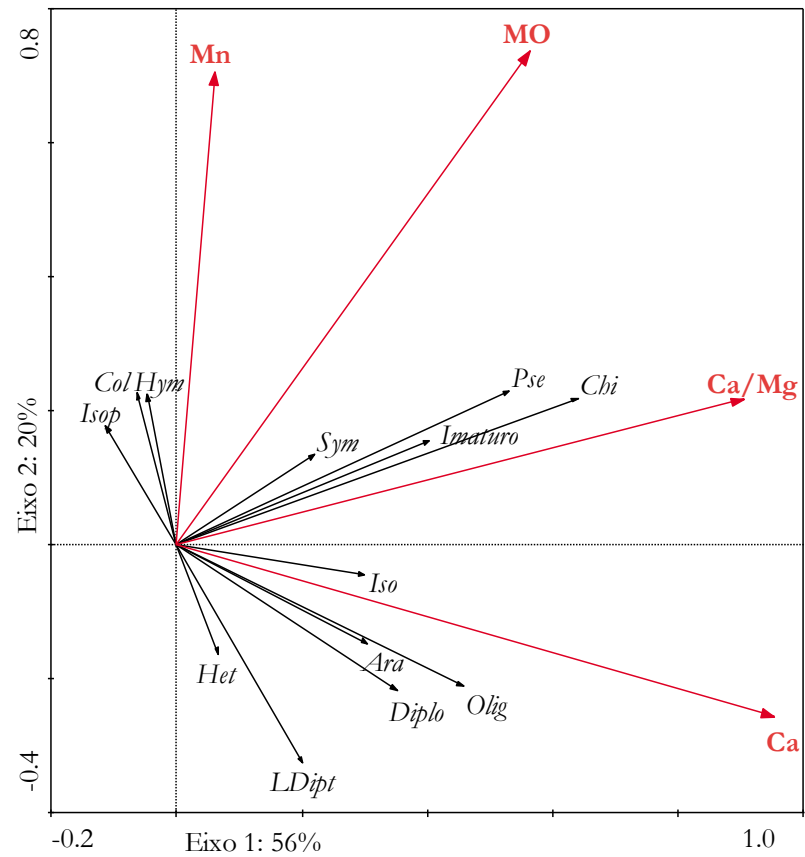

Figura 1. Análise de Redundância (ADR) para comunidade da fauna do solo (flechas pretas) coletadas pelos métodos de Berllese modificado (a) e de monólitos (b) e de alguns atributos químicos e físicos do solo (flechas vermelhas) em setembro de 2011 (primavera). Ver Material e Métodos para legenda dos organismos da fauna do solo e atributos químicos e físicos

De acordo com a ADR (Figura 1a) existiu uma baixa relação entre o teor de Zn com os grupos Pseudoscorpionida, Hemiptera e Collembola. Em estudos com solos contaminados por mineração (BARROS et al., 2010), apontaram que a melhor qualidade ambiental do solo de referência (menores teores de $\mathrm{Zn}$ ) foi atestada pela ocorrência do grupo Pseudoscorpionida somente nesse solo. Nesse estudo apenas a $\mathrm{MN}$, área referência, apresentou ocorrência desse grupo.

De acordo com a ADR (Figura 1a), os atributos $\mathrm{Ca} / \mathrm{Mg}, \mathrm{MO}$ e Umidade foram fortemente relacionados com ocorrência dos grupos Isopoda, Chilopoda, Diptera, Isoptera e Imaturos. Os maiores teores desses atributos foram encontrados na $\mathrm{MN}$, assim como a maior densidade dos grupos. Nesse sentido é provável que haja uma ampla interação entre a biologia e a química do solo, com destaque para esses grupos, que mostraram correlação significativa com os atributos $\mathrm{Ca} / \mathrm{Mg}, \mathrm{MO}$ e Umidade, apresentando-se como potenciais indicadores de qualidade do solo. Possivelmente as práticas agrícolas de manejo do solo e aplicação de resíduos animais provocaram impacto, resultando em redução da ocorrência desses 
organismos nas demais áreas.

A ADR (Figura 1a) apontou relação significativa entre a Ma0-5 cm e os grupos Coleoptera, Hymenoptera, Chilopoda, Diptera e Isopoda. A maior quantidade de macroporos foi encontrada na $\mathrm{MN}$ e M22. Tarrá et al. (2012), concluíram que no grupo de decompositores correlacionados significativamente com o volume dos macroporos do solo, os Isopoda, as Coleoptera e Diplopodas foram responsáveis por 59\% da densidade total de organismos, sendo estes grupos taxonômicos, regularmente mais associados aos processos da biogênese dos poros do solo.

Para o método dos monólitos a ADR, representada na Figura 1b, indicou correlação significativa $(F=6,46 ; \mathrm{p}=0,002)$ com as variáveis ambientais do modelo: $\mathrm{Ca} / \mathrm{Mg}(\mathrm{F}=1,8 ; \mathrm{p}=0,050)$, $\mathrm{Mn}(\mathrm{F}=1,74 ; \mathrm{p}=0,054), \mathrm{MO}(\mathrm{F}=2,56 ; \mathrm{p}=0,001) \mathrm{e}$ $\mathrm{Ca}(\mathrm{F}=6,43 ; \mathrm{p}=0,002)$. Não considerando as variáveis ambientais, o eixo 1 explica $11,4 \%$ do total da variância e o eixo 2 explica 4\%. As variáveis ambientais explicam 20,3\% do total de variação, deste, 56\% é explicado pelo eixo 1 e 19,9\% pelo eixo 2 .

Correspondente com a ADR (Figura 1b), a relação $\mathrm{Ca} / \mathrm{Mg}$ obteve correlação positiva com os grupos da macrofauna Chilopoda, Pseudoescorpionida, Imaturos e Symphyla. O maior teor de $\mathrm{Ca} / \mathrm{Mg}$ ocorreu na MN e para essa área os grupos Chilopoda e

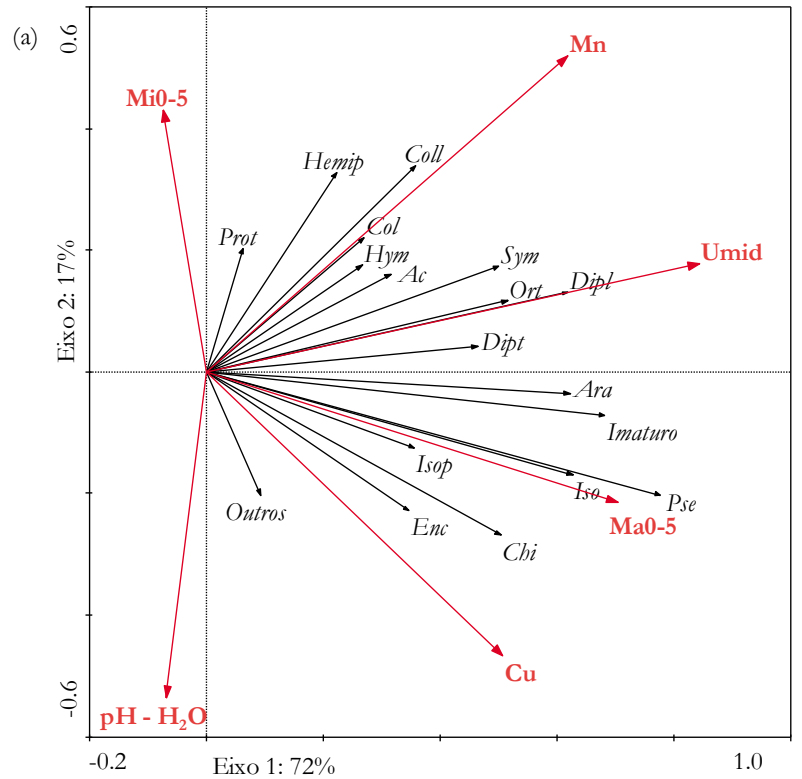

Symphyla obtiveram as maiores densidades, 26 e 6 ind. $\mathrm{m}^{-2}$, respectivamente.

$\mathrm{O}$ atributo MO (Figura 1b) foi correlacionado com os grupos Chilopoda, Symphyla, Pseudoescorpionida e Imaturos. A MN foi a área com maior teor de $\mathrm{MO}(4,46 \%)$ e posteriormente a P22 com $3,67 \%$. Para o grupo Symphyla, a MN e a P22 obtiveram a mesma densidade com 6 ind. $\mathrm{m}^{-2}$. O atributo $\mathrm{Ca}$ foi fortemente correlacionado com Oligochaeta, Diplopoda, Araneae, Isoptera e Larva de Diptera. Observa-se que a densidade de Oligochaeta foi maior para as áreas de MN (111 ind. $\mathrm{m}^{-2}$ ), P5 (62 ind. $\mathrm{m}^{-2}$ ) e M22 (48 ind. $\mathrm{m}^{-2}$ ).

$\mathrm{Na}$ segunda época de coleta (verão), a ADR do método de Berllese modificado (Figura 2a) indicou correlação significativa $(\mathrm{F}=11,42 ; \mathrm{p}=0,002) \mathrm{com}$ as variáveis ambientais do modelo: $\mathrm{Cu}(\mathrm{F}=4,08 ; \mathrm{p}=$ 0,002), $\mathrm{pH}-\mathrm{H}_{2} \mathrm{O}(\mathrm{F}=2,43$; $\mathrm{p}=0,01), \mathrm{Mn}(\mathrm{F}=2,43$; $\mathrm{p}$ $=0,002), \operatorname{Mi} 0-5(\mathrm{~F}=1,77 ; \mathrm{p}=0,058)$, Umidade $(\mathrm{F}=$ $11,42 ; \mathrm{p}=0,002)$ e $\mathrm{Ma} 0-5(\mathrm{~F}=2,99 ; \mathrm{p}=0,002)$. Não considerando as variáveis ambientais, o eixo 1 explica $27,7 \%$ do total da variância e o eixo 2 explica $3,8 \%$. As variáveis ambientais explicam $30,3 \%$ do total de variação, deste $71,5 \%$ é explicado pelo eixo 1 e 16,6\% pelo eixo 2 .

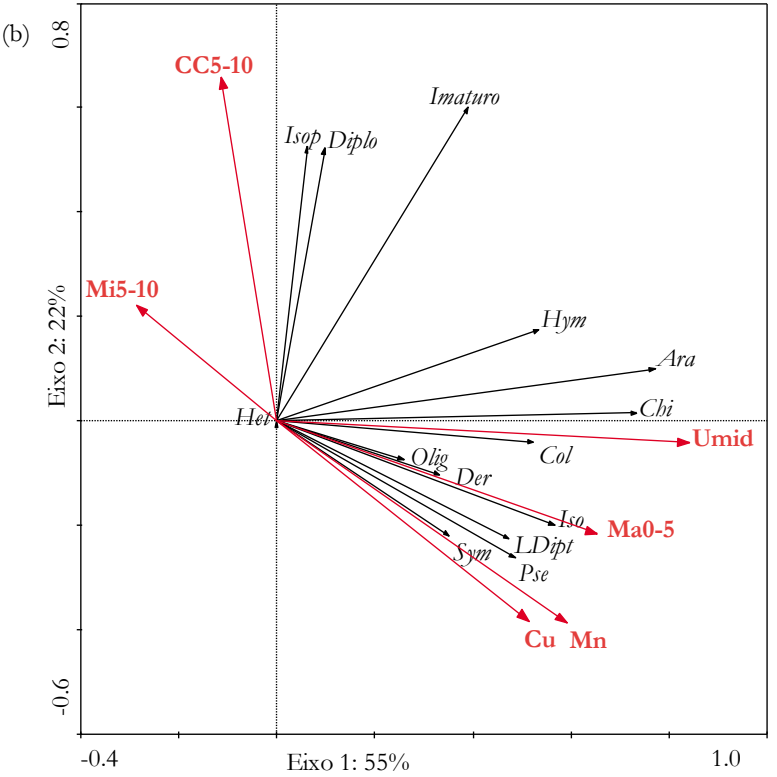

Figura 2. Análise de Redundância (ADR) para comunidade da fauna do solo (flechas pretas) coletadas pelos métodos de Berllese modificado (a) e de monólitos (b) e de alguns atributos químicos e físicos do solo (flechas vermelhas) em dezembro de 2011 (verão). Ver Material e Métodos para legenda dos organismos da fauna do solo e atributos químicos e físicos

De acordo com a ADR (Figura 2a), o Cu apresentou correlação positiva com a ocorrência de
Enchytraeidae e Chilopoda. A maior densidade de Chilopoda foi encontrada na MN, com 51 ind. $\mathrm{m}^{-2}$ e a 
única área com ocorrência de Enchytraeidae, que por sua vez foi o fragmento com maior teor de $\mathrm{Cu}(23,92$ $\mathrm{mg} \mathrm{dm}{ }^{-3}$ ). Segundo Casali et al. (2008), o Cu em solos de mata natural está retido principalmente na $\mathrm{MO}$, e encontraram o valor total de $50,8 \mathrm{mg} \mathrm{dm}^{-3}$ na camada de 0-20. Observa-se na ADR (Figura 2a) uma correlação entre os grupos Isopoda e Pseudoescorpionida e Ma0-5 cm, fenômeno também observado na primeira coleta. A MN apresentou 80 ind. $\mathrm{m}^{-2}$ desse grupo, e a maior quantidade de macroporos.

Conforme a ADR (Figura 2a), observa-se correlação positiva dos grupos Isoptera e Diplopoda com a umidade na CC5-10 cm. Essa correlação é atribuída ao fragmento P22, haja vista ser a área com o maior valor $(41,97 \%)$ da variável CC5-10 $\mathrm{cm}$ e com as maiores densidades para Isoptera (85 ind. $\mathrm{m}^{-2}$ ) e Diplopoda (6 ind. $\left.\mathrm{m}^{-2}\right)$.

$\mathrm{Na}$ ADR (Figura 2a) a correlação positiva de Collembola com o teor de $\mathrm{Mn}$, pode ser pela alta densidade desse grupo (435 ind. $\mathrm{m}^{-2}$ ) associado ao teor de $112,74 \mathrm{mg} \mathrm{dm}^{-3}$ de $\mathrm{Mn}$ na MN. A umidade apresentou correlação positiva principalmente com os grupos Diplura, Larva de Diptera, Symphyla e Ortoptera. Esses organismos alcançaram as maiores densidades na $\mathrm{MN}$, que se encontrava com o maior teor de umidade. Destacando-se o período de estiagem e maior insolação ocorrida no período das coletas, a MN possivelmente por apresentar cobertura vegetal abundante sofreu menor interferência na manutenção da umidade do solo.

Para o método dos monólitos a ADR (Figura $2 \mathrm{~b})$ indicou correlação significativa $(\mathrm{F}=9,78 ; \mathrm{p}=$ $0,002)$ com as variáveis ambientais do modelo: $\mathrm{Mn}(\mathrm{F}$ $=3,31 ; \mathrm{p}=0,012), \mathrm{CC} 5-10(\mathrm{~F}=2,70 ; \mathrm{p}=0,02), \mathrm{Mi0}-5$ $(\mathrm{F}=5,10 ; \mathrm{p}=0,006), \mathrm{Ma0}-5(\mathrm{~F}=2,62 ; \mathrm{p}=0,03)$, Umidade $(\mathrm{F}=9,78 ; \mathrm{p}=0,002)$ e $\mathrm{Cu}(\mathrm{F}=3,74 ; \mathrm{p}=$ $0,002)$. Não considerando as variáveis ambientais, o eixo 1 explica $21 \%$ do total da variância e o eixo 2 explica 8,4\%. As variáveis ambientais explicam 38,1\% do total de variação, deste $55,2 \%$ é explicado pelo eixo 1 e $22 \%$ pelo eixo 2 .

De acordo com a ADR (Figura 2b), os atributos Umidade, $\mathrm{Ma} 0-5 \mathrm{~cm}$, teor de $\mathrm{Mn}$ e $\mathrm{Cu}$ no solo foram correlacionados aos grupos Symphyla, Pseudoescorpionida, Larva de Diptera, Isopoda, Dermaptera, Oligochaeta, Coleoptera, Chilopoda, Araneae e Hymenoptera. Todos esses grupos foram mais abundantes na $\mathrm{MN}$, sendo a área com maior teor de $\mathrm{Cu}$ e $\mathrm{Mn}$ para essa época de amostragem, assim como umidade e Ma0-5. Por ser o fragmento com maior cobertura vegetal manteve a umidade do solo, possibilitando a manutenção da densidade de organismos. Seguinte a MN, a P22 destaca-se para o teor de umidade, com a segunda maior densidade de Chilopoda e Araneae.

$\mathrm{Na}$ terceira época de amostragem (outono), a ADR do método de Berllese modificado (Figura 3a) indicou correlação significativa $(F=4,28 ; \mathrm{p}=0,0002)$ com as variáveis ambientais do modelo: $\mathrm{Cu}(\mathrm{F}=3,46$; $\mathrm{p}=0,002), \mathrm{pH}-\mathrm{H}_{2} \mathrm{O}(\mathrm{F}=2,47 ; \mathrm{p}=0,01), \mathrm{MO}(\mathrm{F}=$ $4,28 ; \mathrm{p}=0,002)$ e $\mathrm{P}(\mathrm{F}=1,90 ; \mathrm{p}=0,056)$. Não considerando as variáveis ambientais, o eixo 1 explica $7 \%$ do total da variância e o eixo 2 explica $5,9 \%$. As variáveis ambientais explicam $17,9 \%$ do total de variação, deste $33,5 \%$ é explicado pelo eixo 1 e $28,8 \%$ pelo eixo 2 .

Em concordância com a ADR, o grupo Diplura mostrou correlação positiva com os atributos Cu e P (Figura 3a) apresentando a maior densidade (66 ind. $\mathrm{m}^{-2}$ ) e os maiores teores de $\mathrm{Cu}$ e $\mathrm{P}$ com 30,27 e $28,87 \mathrm{mg} \mathrm{dm}^{-3}$, respectivamente, observados na P17.

A ADR (Figura 3a) aponta a correlação entre MO e Pseudoescorpionida. O grupo ocorreu apenas na $\mathrm{MN}$, com densidade de 67 ind. $\mathrm{m}^{-2}$. Desta forma, a $\mathrm{MN}$ resultou em 3,87\% de $\mathrm{MO}$, o maior teor comparado com as demais áreas, o grupo Pseudoescorpionida apresentou a maior relação com a $\mathrm{MN}$, assim como nas outras épocas de coleta.

Para o método dos monólitos a ADR, representada na Figura 3b, indicou correlação significativa $(\mathrm{F}=4,08 ; \mathrm{p}=0,004) \mathrm{com}$ as variáveis ambientais do modelo: $\mathrm{MO}(\mathrm{F}=4,08 ; \mathrm{p}=0,004), \mathrm{Zn}$ $(\mathrm{F}=2,33 ; \mathrm{p}=0,002)$ e $\mathrm{K}(\mathrm{F}=1,99 ; \mathrm{p}=0,0018)$. Não considerando as variáveis ambientais, o eixo 1 explica $8,2 \%$ do total da variância e o eixo 2 explica 3,9\%. As variáveis ambientais explicam $14,2 \%$ do total de variação, deste $59,9 \%$ é explicado pelo eixo 1 e $27,2 \%$ pelo eixo 2.

Segundo a ADR (Figura 3b), a variável Zn apresentou correlação positiva com os grupos Oligochaeta e Symphyla. De acordo com Nahmani e Lavelle (2002), o grupo Oligochaeta não suporta concentrações maiores que $2.000 \mathrm{mg} \mathrm{kg}^{-1}$ de $\mathrm{Zn}$. No presente estudo a maior concentração de $\mathrm{Zn}$ foi encontrada na P17 e P5, com 20,73 $\mathrm{mg} \mathrm{dm}^{-3}$ e 16,23 $\mathrm{mg} \mathrm{dm}^{-3}$, respectivamente, ou seja, teores abaixo do valor limitante para ocorrência de Oligochaeta. Compatível com a indicação da ADR, as áreas P17 e P5 obtiveram as maiores densidades de Oligochaeta. 
(a)

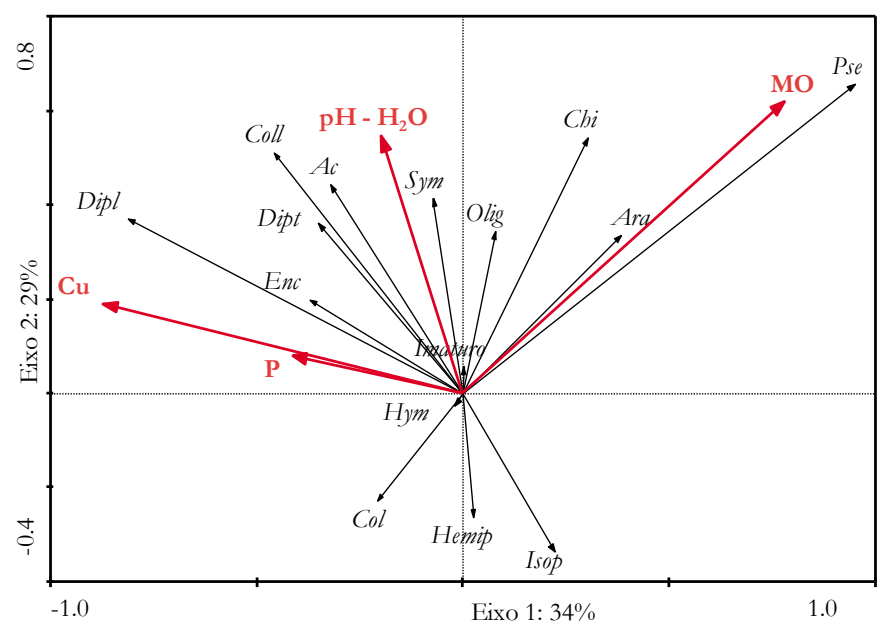

(b)

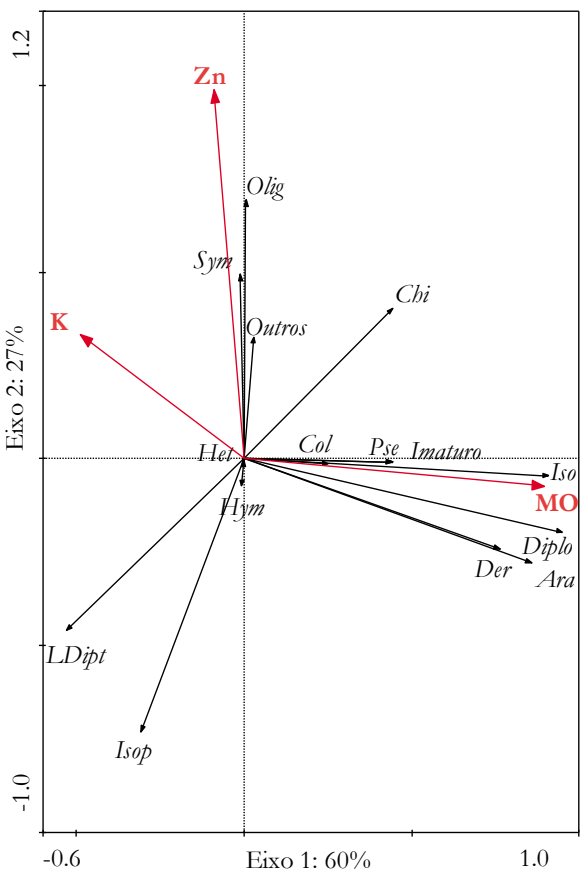

Figura 3. Análise de Redundância (ADR) para comunidade da fauna do solo (flechas pretas) coletadas pelos métodos de Berllese modificado (a) e de monólitos (b) e de alguns atributos químicos e físicos do solo (flechas vermelhas) em março de 2012 (outono). Ver Material e Métodos para legenda dos organismos da fauna do solo e atributos químicos e físicos

$\mathrm{Na}$ quarta época de amostragem (inverno), a ADR do método dos monólitos modificado (Figura 4) indicou correlação significativa $(\mathrm{F}=2,75 ; \mathrm{p}=0,002)$ com as variáveis ambientais do modelo: Ma5-10 (F = $2,75 ; \mathrm{p}=0,002), \mathrm{Cu}(\mathrm{F}=1,94 ; \mathrm{p}=0,018)$ e $\mathrm{pH}-\mathrm{H}_{2} \mathrm{O}$ $(\mathrm{F}=1,75 ; \mathrm{p}=0,038)$. Não considerando as variáveis ambientais, o eixo 1 explica 5,6 \% do total da variância e o eixo 2 explica $3,2 \%$. As variáveis ambientais explicam $11,2 \%$ do total de variação, deste $50,1 \%$ é explicado pelo eixo 1 e $28,5 \%$ pelo eixo 2 .

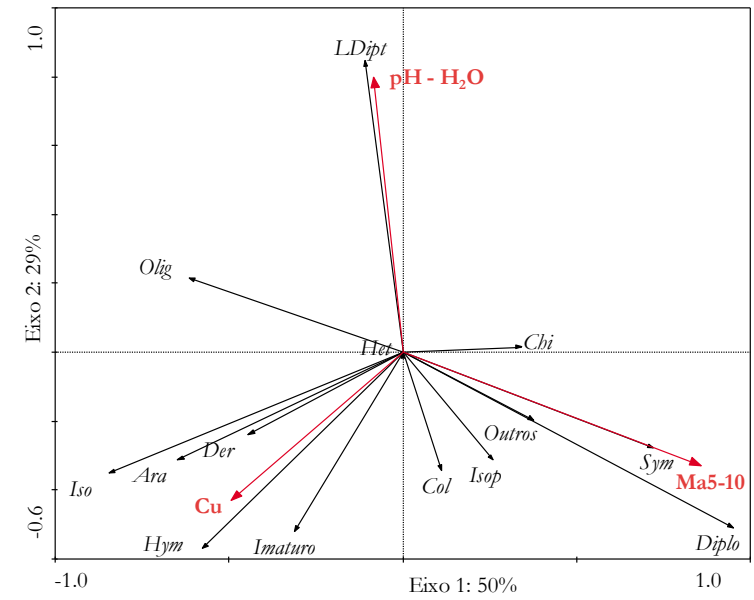

Figura 4. Análise de Redundância (ADR) para comunidade da fauna do solo (flechas pretas) coletada pelo método de monólitos e de alguns atributos químicos e físicos do solo (flechas vermelhas) em junho de 2012 (inverno). Ver Material e Métodos para legenda dos organismos da fauna do solo e atributos químicos e físicos

Em conformidade com a ADR (Figura 4), o atributo $\mathrm{pH}-\mathrm{H}_{2} \mathrm{O}$ apresentou correlação positiva com Larva de Diptera. O grupo larvas de Diptera apresentou a maior densidade de organismos para o inverno de 2012 na área M22, com 120 ind. $\mathrm{m}^{-2}$. A ADR aponta correlação entre a Ma5-10 cm com os grupos Diplopoda e Symphyla. A MN foi o fragmento desse estudo que apresentou a maior quantidade de Ma5-10 (33\%), assim como a maior densidade de Diplopoda e Symphyla.

Além disso, a intensificação da agricultura pode reduzir a biodiversidade do grupo Symphyla, no entanto, pouco se sabe sobre os efeitos dessas transformações no solo para comunidades de Symphyla no Brasil (MORAIS; SILVA, 2009). Pelo fato de migrar verticalmente no solo, a Ma5-10 cm pode ter colaborado com a permanência da comunidade de Symphyla no ambiente, efeito atribuído à MN. 
Análise da curva de resposta principal (CRP) para os grupos da fauna do solo em diferentes métodos de coleta

A CRP é uma técnica multivariada para a avaliação da estrutura das comunidades de grupos ou espécies, o que é adequado para investigar os efeitos estressores (no caso os manejos e tempo de descarte de resíduos orgânicos) sobre todos os grupos da fauna do solo e suas mudanças ao longo do tempo (MOSER et al., 2007).

A análise da CRP referente ao método de coleta pelo Berllese modificado é apresentada na Figura 5a, revelou que $49,9 \%$ da variação total foi explicada pelo eixo canônico 1 (teste de 499 permutações de Monte Carlo; $\mathrm{F}=35,8 ; \mathrm{p}=0,002)$. A variação explicada pelo fator épocas de coleta foi de $10,5 \%$, já os tratamentos explicaram $31,6 \%$ da variação. Ainda, pode-se observar que ocorreu diferença significativa entre a MN (considerada área controle) e as demais áreas do estudo na primavera (exceto P0), verão e outono em P5, M22 e P5. A estiagem prolongada ocorrida no verão pode ser um fator para tal diferença.

(a)
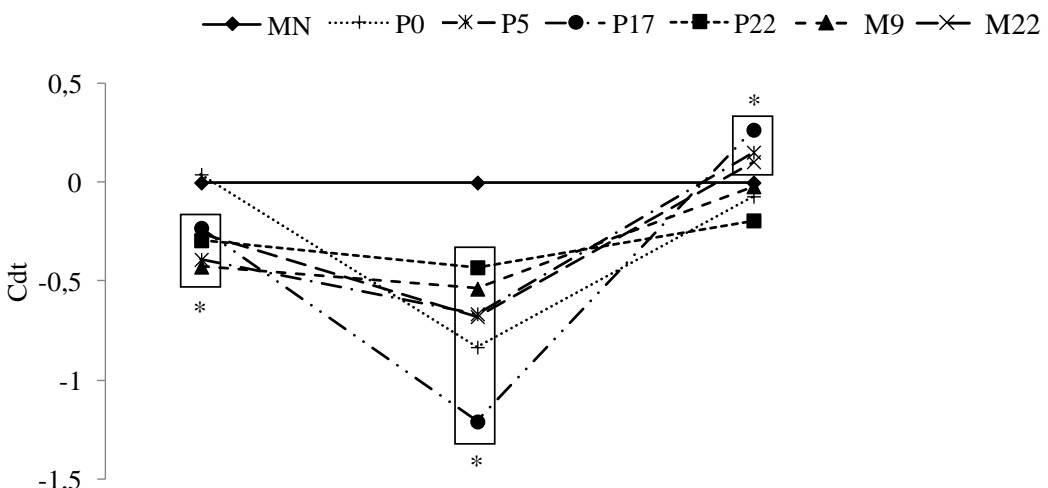

(b)

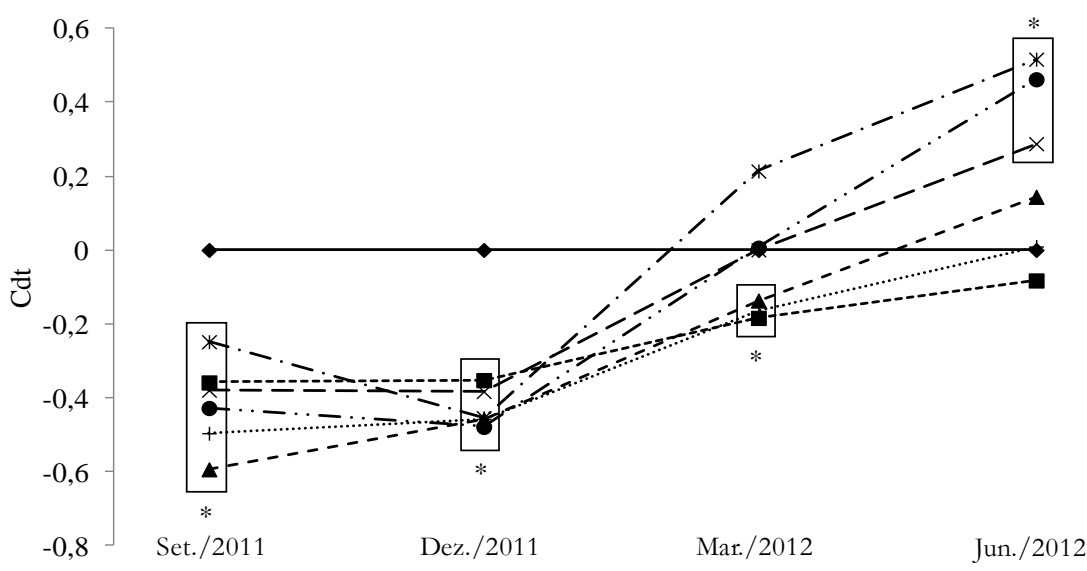

Tempo (épocas de coleta)
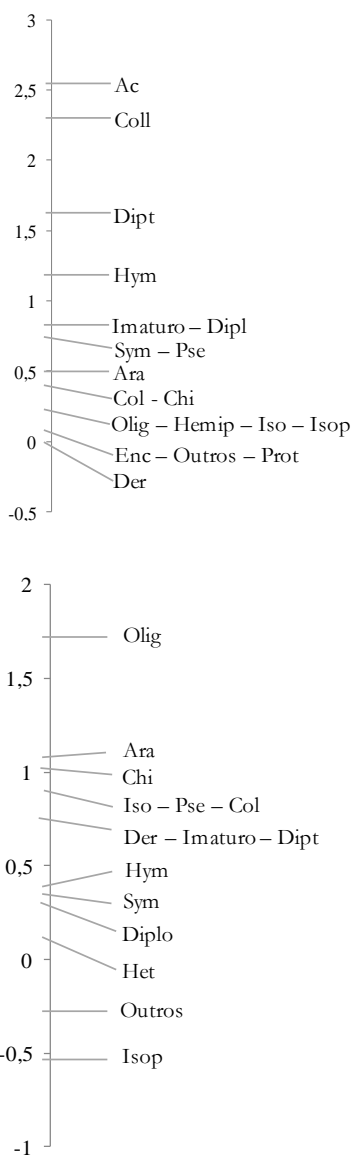

$\mathrm{Bk}$

Figura 5. Curvas de Resposta Principal do efeito dos diferentes manejos e tempos de descarte de resíduos animais (MN, P22, M9, M22, P5, P17 e P0) sobre a densidade de grupos da fauna do solo coletadas pelo método de Berllese modificado (a) e TSBF (b) (Cdt - Coeficiente canônico padronizado da RDA parcial) e o peso dos grupos da fauna do solo (Bk) no município de Concórdia, SC. Ver Material e Métodos para legenda dos organismos da fauna do solo

Através da CRP pode-se observar que os grupos Acarina, Collembola, Hymenoptera e Larva de Diptera, foram grupos com maior peso (Bk), além da densidade mais influenciada pelo manejo em três diferentes épocas de coleta. A MN foi o a área com densidade de organismos mais constante ao longo das estações do ano, seguida da P22 e ao contrário disso, as áreas P0 e P17. Na segunda época de amostragem (verão), foi possível perceber a maior diferença na densidade de organismos e seus pesos entre as áreas do estudo. Os grupos da fauna edáfica capturados pelo método do Berllese, Dermaptera, Protura, Outros e 
Enchytraeidae, apresentaram pouca variação em suas densidades para todas as áreas avaliadas, ao longo das três épocas de coleta, com valores de Bk próximos a 0.

A P22 foi o fragmento mais constante ao longo das coletas, possivelmente por não apresentar revolvimento do solo, pisoteio animal e contar com uma cobertura de pastagem anual perenizada, sofrendo o manejo menos intensivo comparado com os demais fragmentos agrícolas, armazenando maior volume de água no solo em época de estiagem.

A P0 e a P17 em dezembro de 2011, se distanciaram das demais áreas com um decréscimo na densidade e peso dos grupos Acarina, Collembola, Diptera, Hymenoptera e Imaturos, essas áreas são visivelmente afetadas pelo excesso de pisoteio animal que recebem. A M9, P5 e M22 mostraram comportamento semelhante quanto à densidade e peso de organismos ao longo das três épocas de coleta, sendo que no outono obtiveram um aumento na densidade dos grupos Acarina, Collembola, Diptera, Hymenoptera e Imaturos.

A M22 apresentou a segunda maior densidade de Larvas de Diptera, essa ocorrência pode estar relacionada à aplicação de resíduo animal que ocorreu antes da coleta, incentivando a sua proliferação no ambiente. Strieder, Santos e Vieira (2006) em estudo conduzido em áreas de suinocultura e avicultura no Rio Grande do Sul apontam que a densidade do grupo é potencializada pelo descarte de resíduos animais.

A análise da CRP referente ao método de coleta pelo monólito é apresentada na Figura 5b. A CRP revelou que 33,9\% da variação total foi explicada pelo eixo canônico 1 ( $F=20,5 ; p=0,002)$. A variação explicada pelo fator épocas de coleta foi de $7,1 \%$, já os tratamentos explicaram 25,9\% da variação. A partir da CRP, pode-se observar a dinâmica da diferença significativa entre a MN e as demais áreas do estudo no decorrer das quatro épocas de amostragem.

$\mathrm{Na}$ primavera e verão observam-se diferenças significativas entre os sistemas agrícolas em relação ao seu controle $(\mathrm{MN})$. No outono essas diferenças diminuíram, onde apenas M9, P22 e P0 foram significativos. No inverno essas diferenças invertem, ou seja, M22, P5 e P17 foram superiores a MN e as demais áreas não apresentaram diferenças em relação a $\mathrm{MN}$.

Através da CRP da Figura 5b, observa-se que os grupos Oligochaeta, Aranha, Chilopoda e Isopoda foram grupos com maior peso (Bk), com destaque para Oligochaeta, que obteve a densidade mais influenciada pelo manejo em três diferentes épocas de coleta, possível organismo indicador de manutenção da qualidade do solo ao longo do tempo. Na última época de amostragem (inverno) foi possível perceber a maior diferença entre as áreas para a densidade de organismos com maiores pesos. Os grupos Heteroptera e Diplopoda apresentaram pouca variação em suas densidades para todas as áreas avaliadas, ao longo das quatro estações do ano, com valores de Bk próximos a 0 .

Já a P22, por ser uma área com cobertura vegetal perene e sem pisoteio animal, apresenta uma estabilidade na densidade de organismos ao longo do tempo. Esse comportamento pode ser observado no diagrama da CRP desde a primeira época, sendo a segunda área mais próxima da $\mathrm{MN}$ na primeira época de amostragem, já na amostragem de dezembro de 2011, época que ocorreu estiagem, foi o fragmento que mais se aproximou da $\mathrm{MN}$, ou seja, manteve um padrão na densidade de organismos da auna edáfica e assim, dos serviços ecológicos prestados por eles em épocas de 'estresse ambiental'.

As áreas P0, P17, M9, P22, P5 e M22, na primavera e verão, se distanciaram da $\mathrm{MN}$ com um decréscimo na densidade e peso de dos grupos Oligochaeta, Aranha, Chilopoda, Isopoda e Pseudoescorpionida. No outono, essas áreas obtiveram um aumento na densidade desses organismos, alterando o padrão na curva CRP e atingindo menor desvio em relação a $\mathrm{MN}$.

Destaca-se a M22, P5 e P17 no outono, com desvio positivo e se igualando ao controle (MN) no padrão da CRP, respectivamente. Sabe-se que nessa época do ano no sul do Brasil a oferta de forragem é reduzida, utilizam-se feno e silagem produzidos dos excedentes de primavera e verão, a lotação de animais nas pastagens é diminuída por falta da planta forrageira, reduzindo o pisoteio e trânsito de animais no solo durante esses meses, podendo assim, oportunizar o aumento na densidade de Oligochaeta.

No inverno, a P5 e P17 obtiveram o desvio mais positivo em relação a $\mathrm{MN}$ para o padrão CRP, comparando às demais épocas estudadas. Essa situação é atribuída, principalmente, ao aumento na densidade de Oligochaeta nessas áreas. A densidade de Oligochaeta na P5 foi de 195 ind. $\mathrm{m}^{-2}$ e na P17 187 ind. $\mathrm{m}^{-2}$. Possivelmente essas áreas receberam altas concentrações de resíduos animais para obter alta densidade de plantas forrageiras no inverno.

Portanto, a análise multivariada de curva de resposta principal é um instrumento útil para analisar o comportamento da densidade e peso de organismos da fauna edáfica em variados manejos empregados ao solo, podendo ser útil para avaliar a manutenção dos serviços ecológicos prestados ao solo por esses organismos ao longo do ano. 


\section{CONCLUSÕES}

O descarte continuado de resíduos animais no solo ocasiona mudanças no ambiente influenciando as populações da fauna edáfica. Os atributos matéria orgânica e cobre foram variáveis explicativas significativas na Análise de Redundância para as duas metodologias considerando todas as épocas de coleta.

Considerando o efeito da sazonalidade para a fauna edáfica, tanto para mesofauna como para a macrofauna do solo, a densidade de organismos foi alterada de acordo com a estação do ano. O manejo agrícola P22 foi o que obteve a maior constância para densidade de organismos ao longo do tempo. A MN foi eficiente como área de controle para a Curva de Resposta Principal.

Para macrofauna o grupo Oligochaeta obteve a densidade de organismos mais afetada pela sazonalidade. Para a mesofauna Acarina e Collembola foram os grupos com densidade mais influenciada pelas estações do ano. Portanto, foram os organismos que mais determinaram o padrão da Curva de Resposta Principal.

\section{REFERÊNCIAS}

ACCS - Associação Catarinense de Criadores de Suínos. Relatório Anual 2013. Disponível em: http://www.accs.org.br/arquivos_downloads/downloa d.php?arquivo=Relatorio_Anual_ACCS_2013.pdf.

Acesso em: 24 jan. 2013.

AQUINO, A. M.; CORREIA, M. E. F.; ALVES, M. V. Diversidade da macrofauna edáfica no Brasil. In: MOREIRA, F. M. S.; SIQUEIRA, J. O.; BRUSSAARD, L. (Eds.). Biodiversidade do Solo em Ecossistemas Brasileiros. Lavras: Ed. UFLA, 2008. p. 143170.

ASSIS, P. C. R.; SAGGIN JÚNIOR, O. J.; PAULINO, H. B.; STÜRMER, S. L.; SIQUEIRA, J. O.; CARNEIRO, M. A. C. Fungos micorrízicos arbusculares em campos de murundus após a conversão para sistemas agrícolas no Cerrado. Revista Brasileira de Ciência do Solo, v. 38, p. 1703-1711, 2014.

BARROS, Y. J.; MELO, V. D. F.; SAUTTER, K. D.; BUSCHLE, B.; OLIVEIRA, E. B.; AZEVEDO, C. R.; SOUZA, L. C. P.; KUMMER, L. Indicadores de qualidade de solos de área de mineração e metalurgia de chumbo. II - Mesofauna e plantas. Revista Brasileira de Ciência do Solo, v. 34, p. 1413-1426, 2010.
BARROS, E.; NEVES, A.; BLANCHART, E.; FERNANDES, E. C. M.; WANDELLI, E.; LAVELLE, P. Development of the soil macrofauna community under silvopastoral and agrosilvicultural systems in Amazonia. Pedobiologia, v. 47, p. 23-280, 2003.

BIGNELL, D.; CONSTANTINO, R.; CSUZDI, C.; KARYANTO, A.; KONATÉ, S.; LOUZADA, J. N. C.; SUSILO, F. X.; TONDOH, J. E.; ZANETTI, R. Macrofauna. In: MOREIRA, F. M. S.; HUISING, E. J.; BIGNELL, D. E. (Eds.). Manual de Biologia dos Solos Tropicais - Amostragem e Caracterização da Biodiversidade. Lavras: Ed. UFLA, 2010. p. 79-128.

BROMHAM, S.; CARDILLO, M.; BANNET, S.; CARDILLO, M.; BANNET, A. Effects of stock granizig on the ground invertebrate fauna of woodland remmants. Australian Journal of Ecology, v. 24, p. 199-207, 1999.

CASALI, C. A.; MOTERLE, D. F.; RHEINHEIMER, D. S.; BRUNETTO, G.; CORCINI, A. L. M.; KAMINSKI, J.; MELO, G. W. B. Formas e dessorção de cobre em solos cultivados com videira na Serra Gaúcha do Rio Grande do Sul. Revista Brasileira de Ciência do Solo, v. 32, p. 1479-1487, 2008.

CHODAK, M.; NIKLIŃSKA, M. Effect of texture and tree species on microbial properties of mine soils. Applied Soil Ecology, v. 46, p. 268-275, 2010.

EMBRAPA - Empresa Brasileira de Pesquisa Agropecuária. Manual de métodos de análise de solo. Rio de Janeiro: Centro Nacional de Pesquisa de Solos 1997.

EMBRAPA - Empresa Brasileira de Pesquisa Agropecuária. Sistema brasileiro de classificação de solos. $2^{\mathrm{a}}$ ed. Rio de Janeiro: Centro Nacional de Pesquisa de Solos, 2006.

GEE, G. W.; BAUDER, J. W. Particle size analysis. In: KLUTE, A. (Ed.). Methods of Soil Analysis. Part 1. Physical and Mineralogical Methods. Madison: American Society of Agronomy and Soil Science Society of America, 1986. p. 383-411.

KONZEN, E. A. Fertilização de lavoura e pastagem com dejetos de suinos e cama de aves. Disponível em: http://www.cnpms.embrapa.br/destaques/dejetos/Inf ormeTecnicoDejetos.pdf. Acesso em: 24 jan. 2017.

LOURENÇO, K. S.; CORRÊA, J. C.; ERNANI, P. R.; LOPES, L. D. S.; NICOLOSO, R. D. S. Crescimento e 
absorção de nutrientes pelo feijoeiro adubado com cama de aves e fertilizantes minerais. Revista Brasileira de Ciência do Solo, v. 37, p. 462-471, 2013.

MENYAILO, O. V.; HUNGATE, B. A.; ZECH, W. Tree species mediated soil chemical changes in a Siberian artificial afforestation experiment: Tree species and soil chemistry. Plant and Soil, v. 242, p. 171-182, 2002.

MONDARDO, D.; CASTAGNARA, D. D.; BELLON, P. P.; MEINERZ, C. C.; OLIVEIRA, P. S. R.; NERES, M. Adubação nitrogenada da Brachiaria brizantha com dejeto líquido suíno. Revista Brasileira de Agroecologia, v. 4, p. 3265-3269, 2009.

MORAIS, J. W.; SILVA, E. P. Occurrence of Symphyla (Myriapoda) in the region of the Upper Solimões River, Amazonas, Brazil. Pesquisa Agropecuária Brasileira, v. 44, p. 981-983, 2009.

MOSER, T.; RÖMBKE, J.; SCHALLNASS, H. J.; VAN GESTEL, C. A. M. The use of the multivariate Principal Response Curve (PRC) for community level analysis: a case study on the effects of carbendazim on enchytraeids in Terrestrial Model Ecosystems (TME). Ecotoxicology, v. 16, p. 573-583, 2007.

NAHMANI, J.; LAVELLE, P. Effects of heavy metal pollution on soil macrofauna in a grassland of Northern France. European Journal of Soil Biology, v. 38, p. 297-300, 2002.

OLIVEIRA FILHO, L. C. I.; BARETTA, D.; SANTOS, J. C. P. Influência dos processos de recuperação do solo após mineração de carvão sobre a mesofauna edáfica em Lauro Müller, Santa Catarina, Brasil. Biotemas, v. 27, p. 69-77, 2014.

PEEL, M. C.; FINLAYSON, B. L.; MCMAHON, T. A. Updated world map of the Köppen-Geiger climate classification. Hydrology and Earth System Sciences, v. 11, p. 1633-1644, 2007.

SILVA, R. F.; TOMAZI, M.; PEZARICO, C. R.; AQUINO, A. M.; MERCANTE, F. M. Macrofauna invertebrada edáfica em cultivo de mandioca sob sistemas de cobertura do solo. Pesquisa Agropecuária Brasileira, v. 42, p. 865-871, 2007.
SOUTHWOOD, T. R. E. Ecological methods with particular reference to the study of insect populations. London: Chapman \& Hall, 1968.

STRIEDER, M. N.; SANTOS, J. E.; VIEIRA, E. M. Distribuição, abundância e diversidade de Simuliidae (Diptera) em uma bacia hidrográfica impactada no sul do Brasil. Revista Brasileira de Entomologia, v. 50, p. 119124, 2006.

SWIFT, M. J.; BIGNELL, D.; MOREIRA, F. M. S.; HUISING, J. O inventário da diversidade biológica do solo: conceitos e orientações gerais. In: MOREIRA, F. M. S.; HUISING, E. J.; BIGNELL, D. E. (Eds.). Manual de Biologia dos Solos Tropicais - Amostragem e Caracterização da Biodiversidade. Lavras: Ed. UFLA, 2010. p. 23-41.

TARRÁ, I. L. C.; LUIZÃO, F. J.; WANDELLI, E. V.; TEIXEIRA, W. G.; MORAIS, W. J.; FERNANDES, E. C. M.; BROCHEL, K. V.; PÉREZ, N. V. Grupos funcionais da macrofauna e macroporos do solo em sistemas agroflorestais da Amazônia central. Prospectiva, v. 10 , p. $6-17,2012$.

TEDESCO, M. J.; GIANELLO, C.; BISSANI, C. A.; BOHNEN, H.; VOLKWEISS, S. J. Análises de solo, plantas e outros materiais. $2^{\mathrm{a}}$ ed. Porto Alegre: Universidade Federal do Rio Grande do Sul, 1995. (Boletim técnico, 5).

TER BRAAK, C. J. F.; ŠMILAUER, P. CANOCO Reference Manual and CanoDraw for Windows User's Guide: Software for Canonical Community Ordination (Version 4.5). Microcomputer Power, Wageningen. 2002.

UBABEF - União Brasileira de Avicultura. Relatório anual 2014. Disponível em: http://www.ubabef.com.br/files/publicacoes/8ca705e 70f0cb110ae3aed67d29c8842.pdf. Acesso em: 11 jan. 2014.

VAN DEN BRINK, P. J.; TER BRAAK, C. J. F. Principal Response Curves: Analysis of TimeDependent Multivariate Responses of Biological Community to Stress. Environmental Toxicology and Chemistry, v. 18, p. 138-148, 1999. 